Fabio Gatti

\title{
Il «santissimo» Cicerone. La Quaestura di Sebastiano Corradi (1555) nella tradizione biografica sull'Arpinate
}

\section{Introduzione}

Nella premessa della Vita Ciceronis (1415) rivolta a Niccolò Niccoli, Leonardo Bruni auspicava che la propria opera venisse superata da altre più eleganti ed esaustive biografie ciceroniane, perché sul "principe delle lettere» doveva innescarsi un certamen letterario tra dotti. ${ }^{1}$ A distanza di centoquarant'anni la sfida del Bruni sarebbe stata raccolta da Sebastiano Corradi (ca. 1510 - 1556) con la Quaestura, ampia e singolare biografia ciceroniana in forma di dialogo pubblicata a Bologna nel 1555: ${ }^{2}$ in quest'epoca Cicerone aveva a tal punto catalizzato gli interessi dei dotti da essere ormai definitivamente assurto a somma auctoritas, oggetto di una vera e propria venerazione non solo per lo stile, ma anche per l'alto pensiero, ritenuto moralmente edificante e conciliabile con la dottrina cristiana. ${ }^{3}$ Grazie all'influente teorizzazione del cardinale Pietro Bembo, che in Cicerone aveva individuato, nella polemica sull'imitazione con Giovanfrancesco Pico della Mirandola (1512-1513) e nelle Prose della volgar lingua (1525), il modello di prosa latina da assimilarsi e da riprodursi in tutte le sue caratteristiche, il ciceronianismo era riuscito vittorioso dalle periodiche dispute umanistiche sul migliore stile latino, diventando, agli albori della Controriforma, marca identitaria della cultura ufficiale egemonizzata dagli ambienti ecclesiastici, che furono i più ferventi fautori dell'autorità ciceroniana. ${ }^{4}$ L'ammirazione per l'Arpinate si

\footnotetext{
1 Viti 1996, 418.

2 Corradi 1555a. L'autore è registrato in Cosenza 1962-1967, II 1109-1110; per notizie su di lui cf. De Angelis 1983 e Lanzi 2003, 9-12, ma ancora utili, benché datati, Tiraboschi 1781-1786, II 74-86, e Re 1820.

3 Per la fortuna di Cicerone nel Cinquecento cf. Gatti 2017, 113-118, e Gatti 2020, specialmente 24-40; sul secolare processo di appropriazione dell'opera ciceroniana da parte della cultura cristiana, dall'età dei Padri della Chiesa sino all'umanesimo, cf. anche Toffanin 1964; Springer 2018, 23-33, con la recensione di Gatti 2019; Del Giovane 2020.

4 Per le polemiche umanistiche sull'imitazione e il successo del ciceronianismo restano imprescindibili Lenient 1855, Sabbadini 1885 e Scott 1910, ma si vedano anche D’Amico 1984, D’Ascia 1991, 105-159, e Fantazzi 2014; i principali testi della querelle sono raccolti e introdotti in Dellaneva 2007. Per la presenza di Cicerone nella riflessione bembiana si vedano Vela 2001, I
}

Ә OpenAccess. ( 2022 Fabio Gatti, published by De Gruyter. (cc) BY This work is licensed under the Creative Commons Attribution 4.0 International License. https://doi.org/10.1515/9783110748703-011 
era perlopiù sostanziata in emulazione stilistica nell'oratoria della Curia romana, bersaglio polemico del Ciceronianus erasmiano, mentre aveva percorso un versante più professionale a Venezia, grazie all'erudizione degli umanisti e all'attività di Paolo Manuzio, nella quale i lavori ciceroniani (edizioni, commenti, traduzioni) costituiscono oltre il 65\% della produzione tipografica relativa agli autori antichi, e le Epistulae ad familiares rappresentano il titolo in assoluto più frequente nel suo quasi trentennale periodo di attività lagunare $(1533-1561){ }^{5}$ Luogo di congiunzione geografica e ideale tra la capitale della Serenissima e la Roma papalina era Bologna: avamposto settentrionale dello Stato pontificio (nonché sede, tra il 1547 e il 1549, del Concilio temporaneamente trasferito da Trento), la città era però culturalmente legata a Venezia, da dove per tutto il Cinquecento continueranno a provenire i docenti di umanità dell'Alma Mater come Corradi, che vi mantenne la cattedra dal 1544 sino al $1556 .{ }^{6}$ La sua attività riflette in forme esemplari questa pluralità di influenze, perché all'attenta esegesi delle opere ciceroniane, che si riversa anche in commenti di notevole erudizione, si accompagna nella Questura una presentazione intensamente celebrativa della vita di Cicerone.

\section{Tra Venezia e Bologna: Cicerone e Corradi nell'umanesimo contemporaneo}

La stessa Quaestura è legata ai maggiori poli culturali dell'epoca: benché il contesto sia in apparenza integralmente bolognese, non soltanto per il luogo della pubblicazione, ma anche per la dedica ad senatum populumque Bononiensem e per la fisionomia dell'autore, nativo di Arceto, nei pressi di Reggio Emilia, l'opera è in maggior debito con l'ambiente veneziano, dove Corradi si era formato risiedendo nella città lagunare sino al 1540, quando il comune di Reggio Emilia lo reclamò come pubblico docente. Proprio a Venezia, nel 1537, Corradi aveva esordito con la pubblicazione di una prima Quaestura, ${ }^{7}$ che però, in quanto discussione testuale di passi di autori antichi, è un lavoro differente dalla

18 e Vecce 1996; per la definitiva affermazione del modello ciceroniano nel sistema educativo cattolico e protestante cf. Monfasani 2004 e Springer 2018, 101-144.

5 Sulle tendenze ciceroniane della Curia romana e i suoi rapporti con Erasmo cf. l'introduzione di Gambaro 1965, XxI-Xxxi; D’Ascia 1991, 17-38; Gouwens 1993. Sulla presenza di Cicerone nella produzione di Manuzio cf. l'elenco delle edizioni in Renouard 1803, 188-332, e Sterza 2008, 146-148.

6 Cf. Mazzetti 1847, 110; Costa (E.) 1907, 6-9; Sorbelli/Simeoni 1987, II 43; Calcaterra 2009, 247. 7 Corradi 1537. 
Quaestura del 1555 di cui qui si tratta, al di là della comunanza di titolo, cornice dialogica e personaggi. Il legame che Corradi continuò a mantenere con la città lagunare emerge comunque anche dalla seconda Quaestura, a cominciare dall'ambientazione del dialogo, che si immagina svolto a Venezia in uno dei soggiorni che l'autore, ormai ritornato a Reggio Emilia, periodicamente vi compiva, oltreché dai personaggi che interloquiscono con lui, i più anziani umanisti veneti Battista Egnazio, alias Giovanni Battista Cipelli (1478-1553), e Pierio Valeriano, al secolo Giovanni Pietro Bolzani Dalle Fosse (1477-1558). Gli interessi ciceroniani del bellunese Valeriano sono testimoniati da Paolo Manuzio, che nella dedicatoria dell'edizione delle Epistulae ad familiares da lui curata nel 1533 afferma di essersi avvalso del suo contributo. ${ }^{8}$ Nella Quaestura è però l'Egnazio, definito praeceptor meus (p. 88) dal Corradi, a interpretare un ruolo di primo piano, come appare sin dall'intitolazione dell'opera, dove figura il suo nome (Quaestura sive Egnatius), e dalle prime righe, nelle quali Corradi tesse un encomio del maestro, elogiato per la divina memoria e per la straordinaria versatilità intellettuale, che lo rende una sorta di novello Gorgia, capace di discettare di ogni branca del sapere (p. 3-4 e 78). Collaboratore di Aldo Manuzio e del figlio Paolo, corrispondente di Erasmo, che nel suo Ciceronianus lo presenta come vir non minus probus et integer quam eruditus et eloquens, ${ }^{9}$ l'Egnazio fu senz'altro decisivo nell'avviare Corradi a interessi ciceroniani, avendo collaborato alla preparazione e al commento di edizioni aldine di Cicerone, dal De officiis alla Pro Caelio, e avendo dedicato alle Epistulae dell'Arpinate le sue affollate lezioni veneziane del $1531 .^{10}$ In lui gli interessi esegetici su Cicerone si accompagnavano a un'adesione moderata all'indirizzo stilistico teorizzato dal Bembo, suo concittadino e corrispondente: ${ }^{11}$ significativa al riguardo è una responsiva del 1526 nella quale l'Egnazio confessava all'umanista Giovanni Francesco Conti che avrebbe gradito di più le sue lettere se avessero dimostrato una più solerte, ma non esclusiva, imitazione ciceroniana. ${ }^{12}$

8 Cf. Manuzio 1533, p. n. n. Sul Valeriano, figura ampiamente studiata, si vedano i contributi compresi in Pellegrini 2001 e Pellegrini 2004; cf. inoltre Pellegrini 2002 e Rozzo 2004.

9 Gambaro 1965, 224. Per la corrispondenza tra Erasmo e l'Egnazio cf. Allen (P.) 1906-1958, ad indicem.

10 Sull’Egnazio e la sua attività filologica ed editoriale cf. Mioni 1981 e Venier 1993-1996. 11 Dei contatti epistolari tra Egnazio e Bembo è rimasta una lettera inedita inviata dal primo (Vat. Barb. Lat. 2158) e un'altra, scritta dal secondo, compresa in Bembo 1729, IV 230; sul moderato ciceronianismo dell'Egnazio cf. Fera 2003, xxv.

12 Cf. Planerius 1584, 27r.-v.: Illud vero praeterire non possum, quod ab initio debueram, tuas mihi literas multo gratissimas extitisse, quae tamen aliquanto plus iucunditatis attulissent, si ingenii tui felicitatem ad meliora vertisses. Hoc est, si te Ciceronis, aut eius aetatis scriptorum, similiorem esse maluisses. 
La presenza di Cicerone è centrale nella biografia del Corradi, e sembra in qualche modo orientare anche la scelta dei suoi contatti, a partire dal Bembo, da lui definito vir clarissimus familiaris nella Quaestura (p. 10): in una lettera del 1538 indirizzata all'alto funzionario urbinate Pietro Panfilo, Bembo segnala come precettore per Giulio della Rovere, figlio del duca di Urbino, «Sebastian Corrado da Reggio prete molto dotto in Lattino e convenevolmente in Greco», ${ }^{13}$ rilevandone, oltre allo status ecclesiastico, la conoscenza di entrambe le lingue classiche; in un'altra epistola dello stesso anno, ${ }^{14}$ Corradi sottopone al Bembo, apostrofato come doctorum hominum huius aetatis coryphaeus, l'interpretazione del grecismo ßoũrıৎ di Cic. Att. 2.9.1 come maliziosa allusione a Clodia, che, tacciata di una relazione incestuosa con il fratello Clodio, è degna di essere paragonata alla «boopide» Giunone, sorella e moglie di Giove: l'interpretazione, in effetti corretta, ottenne l'autorevole approvazione del Bembo. ${ }^{15}$ Altro importante contatto veneziano del Corradi fu Paolo Manuzio, con il quale, anche dopo il trasferimento a Reggio Emilia nel 1540 e poi a Bologna dal 1544, egli mise a frutto le lezioni dell'Egnazio nell'emendazione di passi ciceroniani; ${ }^{16}$ con l'editore Corradi manterrà per tutta la vita un rapporto confidenziale, tanto da prodigarsi invano, nel 1555, perché trasferisse la stamperia a Bologna, e da essere da lui affettuosamente definito «compare» in una lettera dell'anno successivo. ${ }^{17} \mathrm{Gli}$ esiti dell'esegesi ciceroniana del Corradi videro la luce sul finire degli anni '30, e poi, soprattutto, tra anni '40 e '50: già la prima Quaestura garantì al suo autore l'ammirazione, secondo quanto lo stesso Corradi racconta nell'omonima opera posteriore (p. 8-10), non soltanto dei maggiori esponenti dell'umanesimo italiano, ma anche di intellettuali europei come l'editore basileano Johannes Oporinus, che nel 1556 rieditò la seconda Quaestura. ${ }^{18}$

Il debito del Corradi nei confronti dell'umanesimo veneziano è del resto comprovato dal fatto che nel 1540 egli accettò l'incarico di docenza nella città natale, Reggio Emilia, solo grazie alle sollecitazioni dell’Egnazio, quando avrebbe invece preferito trattenersi nella città lagunare per continuare a godere

13 Travi 1993, IV 143. La conoscenza del greco del Corradi è testimoniata da Corradi 1543, traduzione latina di sei dialoghi platonici.

14 Cf. Mercati 1951-1982.

15 Cf. sul passo Shackleton Bailey 1965-1970, II, 369.

16 Corradi 1537, 38: Eodem tempore ego Regii, ille [scil. Manutius] Venetiis, multa Ciceronis loca eodem modo correximus.

17 Cf. Manuzio 1556, f. 25r.-v. (lettera di P. Manuzio a Carlo Sigonio datata Bologna, 10 agosto 1555) e ff. 114r.-115r. (lettera di P. Manuzio a S. Corradi datata Venezia, 1 febbraio 1556).

18 Corradi 1556. 
della frequentazione dei massimi dotti del tempo: ${ }^{19}$ tra questi Pier Vettori, editore tra il 1534 e il 1537 dell'intero Cicerone presso i tipi veneziani di Lucantonio Giunta e suo commentatore, al quale Corradi indirizzò una lettera nel 1542 in cui lamentava la difficoltà di coltivare a distanza l'amicizia con lui, vir literatissimus et eruditissimus. ${ }^{20}$ L'insegnamento reggiano, dominato, come il periodo di apprendistato veneziano, dall'esegesi ciceroniana, si concretizzò nel 1544 in un commento alle Epistulae ad Atticum pubblicato non a caso ancora a Venezia e aperto, oltre che da una dedica al cardinale Alessandro Farnese, da una pre-

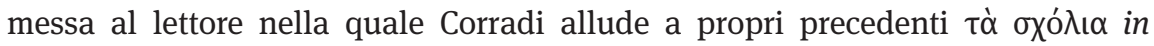
epistolas, quae Familiares dicuntur ${ }^{21}$ di cui è rimasta testimonianza in una miscellanea di annotazioni alle Familiares, comprendenti anche quelle del Vettori, edita nel 1540 a Basilea. ${ }^{22}$ Il commento sistematico alle Epistulae ad Atticum viene presentato dal Corradi come particolarmente gravoso, tanto che, come si fa notare, i dottissimi amici Pier Vettori e Paolo Manuzio si erano limitati rispettivamente a sparse osservazioni filologiche e a sintetici scolii. ${ }^{23}$ Il lavoro gli era stato però richiesto da Romolo Amaseo (1489-1552) e da Marcantonio Flaminio (1498-1550), entrambi desiderosi che il materiale raccolto per le sue lezioni reggiane fosse reso noto al pubblico: ${ }^{24}$ il primo, predecessore del Corradi sulla cattedra bolognese di umanità (1538-1544), era infatti oratore di stretta osservanza ciceroniana e strenuo propugnatore della superiorità del latino sul volgare; il secondo è introdotto come fervente ciceroniano nel Cicero revocatus et Cicero relegatus. Dialogi festivissimi, curioso dialogo dell'umanista milanese Ortensio Lando del 1534, nel quale viene inscenata una dissacrante disputa sull'Arpinate, dapprima condannato all'esilio in quanto reo di ogni vizio morale

19 Cf. Corradi 1555a, $10-11$.

20 Bandini 1758, I 19-20. Per gli interessi ciceroniani del Vettori cf. Mouren 2009; Mouren 2010; Accame 2018.

21 Corradi 1544, f. 3r.

22 Vettori 1540. Ė invece priva di riscontri un'edizione del 1537 riferita in Tiraboschi 1781-1786, 83.

23 Cf. Corradi 1544, f. 3r.: Amicissimi mei Petrus Victorius et Paulus Manutius, viri doctissimi et de literis latinis optime meriti, plurimum operae et studii in eam rem contulerint, quia tamen neuter illorum totum opus exposuit, sed alter castigationibus, ut vocat, alter oxólıoı et his admodum paucis contentus fuit. Le due opere evocate sono, rispettivamente, Vettori 1540 e Manuzio 1540. 24 Cf. Corradi 1544, f. 3r.: Illud dico, M. Antonium Flaminium et Romulum Amasaeum, viros optimos et homines doctissimos, mihique summa benevolentia coniunctos, superioribus mensibus Regium quum venissent, Pontificem Max[imum] comitati, mihi auctores fuisse, ut, quae intellexissent a me in Epistolis ad Atticum interpretandis explicata, ea, foras dari sinerem. Sulla figura e gli interessi culturali di Flaminio cf. Pastore 1981; per Romolo Amaseo cf. Avesani 1960 e Billanovich/Frasso 1979. 
e letterario, ma poi richiamato in patria grazie al solerte intervento di personaggi che ne ristabiliscono la statura intellettuale..$^{25}$

Nella premessa al commento ai sedici libri delle Epistulae ad Atticum, Corradi cita le parole di Cornelio Nepote (Att. 16.3) per evidenziare una peculiarità dell'epistolario ciceroniano che lo renderà del tutto adatto a essere sfruttato nella Quaestura come fonte biografica e storica, ossia il suo contenere lettere scritte in più di vent'anni, dal consolato di Cicerone fino alla sua morte, cosicché «chi le legge non rimpiange più di tanto una storia sistematica di quei tempi» (qui legat, non multum desideret historiam contextam eorum temporum): del resto l'intento di abbozzare una storia della Roma tardorepubblicana attraverso l'epistolario sembra essere appartenuta a Cicerone stesso, che vi volle includere anche lettere ricevute da personalità terze. ${ }^{26} \mathrm{Il}$ commento all'opera si avvale anche di lavori precedenti, rispetto ai quali Corradi annuncia, diversamente da quanto aveva fatto per gli scolii alle Familiares, di dichiarare costantemente il proprio debito per rendere giustizia a quei pochi eruditi che hanno saputo illuminare aspetti di un'opera quanto mai complessa e perciò tra le più trascurate. ${ }^{27}$ Le figure menzionate sono ancora Pier Vettori e Paolo Manuzio, ma anche Andreas Cratander (Andreas Hartmann), prolifico editore riformato che nel 1528, a Basilea, stampò gli opera omnia ciceroniani; ${ }^{28}$ a loro viene riconosciuto il merito di essersi dedicati all'epistolario ad Attico e di averlo emendato per quanto possibile, benché il risultato sarebbe stato più soddisfacente se essi avessero potuto basarsi su un maggior numero di manoscritti rispetto ai due soli codici autorevoli, ancorché corrotti e guasti, sopravvissuti all'epoca: l'uno, che si diceva fosse appartenuto al Petrarca (in realtà un apografo di un suo manoscritto), nelle mani del Vettori; l'altro, noto all'umanista alsaziano Beato Renano, impiegato dal Cratander. ${ }^{29}$ Nel congedarsi dal lettore, Corradi insiste

25 L'opera è ora tradotta e commentata in Tinelli 2017, con informazioni e bibliografia sull'autore alle p. 3-21; per l'orientamento ciceroniano del Flaminio cf. p. 109.

26 Cf. Corradi 1544, f. 3r.: Et sane Cicero historiam quasi contexere videtur voluisse, quum epistolas non solum suas, sed alienas etiam in haec volumina referendas curarit.

27 Corradi 1544, f. 3v.: Nullius scriptoris nomen, quod in nostris Familiarium oxó入ııь scimus desideratum, tacuimus. A primo quidem non ita cogitaram, ut minima quaeque quasi interpres persequerer, sed me postea et rei susceptae amor, et communis utilitas movit, ut nihil fere, de quo vel dubitari posset, praetermitterem, praesertim si ab aliis aliter vel descriptum, vel interpretatum fuisset. Videmus nullum esse librum, in quo tam librarii quam interpretes minus operae et studii posuerint.

28 Cratander 1528; su Cratander editore di Cicerone cf. Canfora 1996.

29 Cf. Corradi 1544, f. 3v.: Semper tamen excipio Andream Cratandrum, Petrum Victorium et Paulum Manutium, qui pro virile laborarunt, ut librum nobis hunc quam emendatissimum traderent: quod illi, quae fuit illorum diligentia, essent assecuti, si plures libros manu scriptos ha- 
sull'obiettivo del lavoro, ossia la chiarificazione dei Realien che si affollano nell'epistolario ciceroniano, talmente fitto di rimandi a leges, plebiscita, senatusconsulta, Praetorum edicta da essere l'opera latina di gran lunga più complessa.

L'interesse per gli aspetti storici, sociali e istituzionali presupposti dalle opere antiche rappresenta un'acquisizione tipica dell'umanesimo veneziano cinquecentesco, che, ben digerita la fase di ammirazione estatica dei classici, vi affianca una più matura attività di contestualizzazione erudita. Nel Corradi tale aspetto si ritrova nel suo secondo lavoro ciceroniano, un commento al Brutus pubblicato a Firenze nel $1552 .{ }^{30}$ L'opera, edita dallo stampatore olandese Lorenzo Torrentino (Laurens van den Bleeck, 1499-1563), che Corradi aveva probabilmente conosciuto a Bologna prima che quegli si trasferisse a Firenze nel $1546,{ }^{31}$ è aperta come di consueto da una dedica (datata primo giugno 1552) indirizzata a una personalità dell'alta società del tempo, in questo caso il parente e conterraneo Sebastiano Antonio Pighini (1500 -1553), padre conciliare di cui si saluta la fresca nomina cardinalizia. ${ }^{32}$ Più interessante, per comprendere retroterra e propositi del lavoro, è però la premessa al lettore, dalla quale emerge il legame sempre stretto tra le pubblicazioni e l'attività di docenza del Corradi, dal 1544 definitivamente approdato allo Studio bolognese: qui, dopo aver inaugurato le lezioni con un discorso di natura pedagogica sui rispettivi doveri del docente e dei discenti pubblicato dal Torrentino, ${ }^{33}$ tenne un corso sul Brutus.

Presentando l'opera come la riproduzione pressoché integrale di sue lezioni tenute per tre anni, Corradi riferisce di avere atteso per due anni un giudizio, prima di pubblicarla, non soltanto ${ }^{34}$ da studiosi dell'antichità, ma anche dai

buissent; sed quum duo tantummodo huiuscemodi libri, qui auctoritatis habeant, hodie reperiantur, alter in Italia apud Petrum Victorium, qui Francisci Petrarchae fuisse dicitur, alter in Germania, quem Beatus Rhenanus legit et Cratander videtur secutus, et hi quoque, ut apparet, dimidiati et corrupti, ex his difficile esset, vel potius plane ádúvatov, ita omnia restituere, ut nihil desiderares. Su identità e vicende del primo codice, il Laurentianus Pluteus 49, 18, cf. Rizzo 1991. 30 Corradi 1552. Il lavoro è preceduto nel 1545 da un'edizione commentata di Valerio Massimo più volte riedita in Italia e all'estero: cf. Corradi 1545.

31 Sul Torrentino cf. Slits 1995.

32 Su Pighini cf. Ceccarelli 2015; la dedicatoria del Corradi è tradotta e commentata in Agosti 1987.

33 L'opera è tradotta e commentata in Lanzi 2003.

34 Corradi 1552: p. n. n. (=4a della premessa al lettore): Volumus enim te [scil. lectorem] nunc omnium, quae in hoc commentario scripsimus, esse iudicem, quamvis ipse scire possis [...] nos ea treis annos totidem paene verbis, quot edita sunt, in hoc nobilissimo Gymnasio Bononiensi exposuisse, deinde duos annos, Apellis exemplo, quid de his homines iudicarent, expectasse, donec ita iudicarunt omnes, quicunque nos audiebant aut literas sciebant, ut ipsi nec possimus nec velimus ad alios iudices appellare. 
maggiorenti della città (principes civitatis), membri dell'alta aristocrazia ecclesiastica e del consesso tridentino (da Giovanni Angelo Medici, futuro papa Pio IV, al delegato pontificio Camillo Mentuati, da Marcello Crescenzi a Girolamo Sauli), tra i quali spicca il nome dell'influente cardinale Giovanni Morone (15091580): ${ }^{35}$ sembra infatti che proprio a quest'ultimo, legato apostolico a Bologna tra il 1544 e il 1548, Corradi dovesse l'ottenimento della prestigiosa cattedra bolognese, stando almeno a quanto lui stesso afferma nella dedica, indirizzata al Morone, del proprio ultimo lavoro, un commento al primo libro dell'Eneide pubblicato ancora dal Torrentino nel $1555 .{ }^{36} \mathrm{Il}$ riferimento rappresenta una significativa testimonianza del fatto che all'epoca l'interesse per Cicerone non era relegato a ristrette cerchie di specialisti, ma costituiva una sorta di comune denominatore di tutti i colti, e in particolare degli ambienti ecclesiastici; è proprio la sua presenza nel curriculum, infatti, a rappresentare per uno studioso dell'epoca la migliore credenziale per ambire a cattedre di rilievo, tanto più agli occhi di personalità come Morone, introdotto come personaggio decisamente ciceroniano nei citati Dialogi del Lando: in quest'opera, infatti, il futuro cardinale perorava con veemenza la causa dell'Arpinate, tessendone un incondizionato elogio, facendone, nel quadro di consolidate riflessioni risalenti almeno al Petrarca, un antesignano del messaggio cristiano («per quanto mi riguarda, tutte le volte che leggo Cicerone mi sembra di leggere non un esperto di diritto, non un retore, ma un uomo cristianissimo, un esimio araldo del Vangelo»), e concludendo che nulla vi fosse di meglio per la formazione di un giovane che la lettura dell'opera ciceroniana. ${ }^{37}$

La pacifica conciliazione di fede religiosa e interessi ciceroniani, avvertita in tutte le sue problematiche implicanze almeno sin dal celebre sogno di san Girolamo (epist. 22.30), era all'epoca preoccupazione particolarmente viva negli ambienti ecclesiastici ai quali Corradi si dichiara vicino, soprattutto dopo le aspre polemiche innescate dal Ciceronianus di Erasmo, che aveva ravvisato un

35 Sul Morone cf. l'ampio Firpo/Maifreda 2019.

36 Cf. Corradi 1555b, 7: Haec vero, quae nos ipsi scripsimus, tamquam tua munera cape, quae tibi Corradus tuorum beneficiorum inde mittat ubi tu, quae tua summa fuit humanitas, eum Bononiae, quum legatus esses, collocasti.

37 Cf. Tinelli 2017, rispettivamente 87 (ego certe quotiescunque illum [scil. Ciceronem] lego, non iuris consultum, non rhetorem aliquem sed christianissimum hominem, sed eximium quendam Evangelii praeconem videor legere) e 89 (itaque sic statuo [...] non posse melius suas horas collocare studiosum iuvenem, quam si illas Cicerone legendo impenderit). La piena conciliabilità del pensiero ciceroniano con la dottrina cristiana è asserita a chiare lettere già dal Petrarca in fam. 21.10.13: Si [sc. Cicero] vidisset Cristum aut nomen eius audivisset, quantum ego opinor, non modo credidisset in eum sed eloquio illo incomparabili Cristi preco maximus fuisset. Per la ricezione di Cicerone in Petrarca cf. Feo 2006 e McLaughlin 2015. 
malcelato paganesimo nell'idolatria di cui l'opera e la figura dell'Arpinate venivano fatte oggetto nel cattolicesimo italiano. ${ }^{38}$ Echi di tali polemiche si riverberano anche negli scritti del Corradi, in particolare nella Quaestura, dove un vivace scambio di battute con l'Egnazio rivela la volontà dell'autore di scagionarsi dall'accusa di essere «poco cristiano» per la scelta di dedicarsi allo studio dell’antichità pagana (p. 16):

\begin{abstract}
Egn.: Scimus te diu multumque in veterum rebus esse versatum, neque id ipsum sine reprehensione. Cor.: Qui me tandem reprehendunt? Egn. Qui suum studium in philosophia, vel in iure civili, vel in sacris litteris collocarunt. Cor.: Quid me reprehendunt? Egn.: Christianum hominem tantum temporis hisce rebus perquirendis consumere. Cor.: Quasi vero parum, vel nihil omnino, iuvet hominem Christianum, quum quae iactant isti, quantum satis est, norit, historias quoque, quas maiores nostri prorsus ediscebant, nosse; aut iam convicerint ipsi, Christianos esse magis, qui terrarum orbem captionibus, litibus et erroribus replent ac perturbant, quam qui historias ita legunt, ut interea tamen vere philosophentur, leges servent et Deum colant?
\end{abstract}

Egn.: Sappiamo che tu ti sei dedicato molto e a lungo alle questioni di antichità, e ciò non senza rimproveri. Cor.: Chi mi rimprovera? Egn.: Chi ha rivolto il proprio impegno alla filosofia, al diritto civile o alle sacre scritture. Cor.: Che cosa mi rimproverano? Egn.: Che un cristiano spenda tanto tempo nello studiare queste cose. Cor.: Come se davvero al cristiano giovasse poco o nulla, quando ha imparato quanto basta le cose a cui si dedicano costoro, studiare anche la storia che i nostri avi imparavano addirittura a memoria; o ormai ci avranno convinti che sono più cristiani proprio loro, che riempiono e turbano la terra con inganni, liti ed errori, di quanti studiano la storia in modo tale che nel frattempo fanno davvero filosofia, onorano le leggi e venerano Dio?

Protestando la piena compatibilità dei propri studi con l'identità cristiana, Corradi pone l'accento sulla massima utilità della storia antica per l'epoca presente, replicando alle accuse, già rintuzzate nella premessa del commento al Brutus del 1552, secondo le quali un uomo del suo tempo avrebbe fatto meglio a dedicarsi ad altro genere di studi. ${ }^{39}$ Nelle parole del Corradi si riflettono invece le convinzioni degli ambienti ecclesiastici e della cultura ufficiale dell'epoca, che sotto l'influsso della temperie umanistica continuavano a considerare l'antichità, e il suo massimo rappresentante Cicerone, come summa di ogni sapere:

38 Sul tema cf. D'Ascia 1994, 173-207.

39 Cf. Corradi 1552, p. n. n. (= III della premessa al lettore): Et sane quidem nos ita semper utilitati communi studuimus, ut, ea contenti, quum res alias, tum vero vel in primis quorundam calumnias neglexerimus qui soleant eos, qui res huiuscemodi paulo diligentius perscrutentur, accusare, quasi vero convicerint eas aut hodie nihil prodesse aut a magnis viris olim spretas fuisse; quum satis appareat contra, nihil esse, quod studiosis hominibus magis prosit, nihil, in quod magni viri plus studii videantur contulisse. 
emblematica, in proposito, sarà, meno di dieci anni dopo, la scelta dei padri tridentini di assegnare l'orazione conclusiva del Concilio a un giovane vescovo veneziano, Girolamo Ragazzoni (1537-1592), che a soli ventisei anni non poteva vantare particolari meriti pastorali, ma che già si era segnalato per un commento latino alle Epistulae ad familiares e un volgarizzamento delle Filippiche ciceroniane, pubblicati intorno alla metà degli anni '50 da Paolo Manuzio. ${ }^{40}$

Il solido legame del Corradi con gli ambienti ciceroniani emerge anche laddove l'autore riconduce la genesi del commento al Brutus alle sollecitazioni del bresciano Giovita Ravizza (Rapicius, 1476-1553), dal 1523 rinomato docente a Venezia e autore di un trattato sul ritmo della prosa oratoria, il De numero oratorio, pubblicato postumo da Paolo Manuzio nel 1554.41 Ravizza legherà il proprio nome a Cicerone a tal punto che il suo testamento sarà pubblicato insieme ad altri, nel 1581, nel commentario aldino al De officiis, opera su cui egli aveva tenuto alcuni corsi veneziani. ${ }^{42}$ A lui Corradi aveva sottoposto la bozza della Quaestura del 1537, ottenendo un giudizio lusinghiero e ricevendo l'invito a comporre un volume prosopografico de personis Ciceronianis volto a fornire informazioni sui personaggi che si affollano nell'opera dell'Arpinate; l'utilità dell'operazione viene condivisa dallo stesso Corradi, che, accantonato l'iniziale scetticismo dovuto alla gravosa mole del lavoro, ritiene che gli studiosi vi potranno trarre non poco giovamento, ancor più di quello prodotto dai già esistenti studi prosopografici sulle personae oraziane, figure perlopiù di scarsa importanza..$^{43}$ La scelta di dare seguito alle esortazioni di Ravizza concentrandosi sul Brutus, pur senza prescindere dall'attenta lettura di Ciceronis omnes libros, è giustificata dal Corradi con la constatazione che in quell'opera, più che in ogni altra, si concentrano, «come in un cavallo di Troia», così tanti oratori greci e latini che «chi conosce bene quelli, può ritenere di conoscere quasi tutti i per-

40 Sull'argomento cf. Gatti 2017, $118-122$.

41 Ravizza 1554. Su Ravizza cf. ancora Boldrini 1903 e l'aggiornato Valseriati 2016.

42 Cf. Manuzio 1581, 136-138.

43 Cf. Corradi 1552, p. n. n. (= I della premessa al lettore): Quum Iovita Rapicius, vir optimus et homo doctissimus, Quaesturam nostram ne adhuc quidem descriptam legisset, nos, ut de personis Ciceronianis librum scriberemus, hortatus est [...] Iam tum viro prudentissimo parere constituimus, praesertim quum certo scieremus, eum, qui diligenter id fecisset, multo plus utilitatis, quam qui personas Horatianas collegerunt, studiosis hominibus allaturum. Nam si maiores nostri eos, qui personas poetae nec summi nec multas nec admodum illustres, quae tamen adhuc multis essent notae, descripserunt, multum profuisse putaverunt, quantum nos eum, qui personas historici, philosophi et certe summi oratoris innumerabiles et olim quidem illustres, sed nunc maiorem partem propter rerum ignorationem obscuras, rursus illustret scriptis profuturum putare debemus? 
sonaggi ciceroniani». ${ }^{44}$ Già nel commento alle lettere ad Attico Corradi si era posto il problema di identificare sul piano storico le personalità menzionate dall'Arpinate, ma in quel caso aveva confessato di lasciare volentieri ad altri l'identificazione di nomi ignoti e perlopiù trascurati anche dai precedenti studiosi, specialmente quando si trattava di figure marginali..$^{45} \mathrm{Nel}$ caso del Brutus l'identificazione storica delle numerosissime personalità citate diviene invece necessaria, trattandosi di illustri oratori, assertori di specifiche teorie retoriche; essa costituirà un motivo di orgoglio per Corradi, deciso a segnare non senza polemica un contrasto con i precedenti esegeti ciceroniani, rei di riproporre confusamente concetti già ampiamente noti o non pertinenti, senza contribuire al benché minimo progresso negli studi sul massimo esponente della latinità e sulla sua epoca:

Interpretes enim, si quid occurrat historiae, solent, quod «lippis et tonsoribus est notum» [Hor. serm. 1.7.3] et a re saepius alienum, referre; is [sc. meus liber] nihil, nisi quod reconditum sit, et ad rem maxime pertineat, edendum curabit. Illi, si M. Cato, M. Piso, L. Torquatus, L. Lucullus aliquid apud Ciceronem de philosophia disputabunt, multa quidem congerent, sed ita confundent, ut ea nec caput, nec pedes habere videantur; is vero, cur Cato, Piso, Torquatus, Lucullus potius, quam Varro, Cotta, Balbus, Triarius et cur eo modo potius, quam alio loquantur, quam planissime fieri poterit, explicabit [...] Quid multa? Is in Ciceronis operibus, quicquid ad historiam, quicquid ad philosophiam, quicquid ad artem dicendi pertinet, ita nobis, si modo studii tantum, quantum requiritur, in eam rem conferre volet, explanabit, nihil ut amplius desiderare possimus. ${ }^{46}$

Infatti gli interpreti, se capita qualche questione di storia, sogliono riferire ciò che «è noto ai cisposi e ai barbieri» [Hor. serm. 1.7.3] ed è molto spesso estraneo al tema; questo libro, invece, non si occuperà di nulla se non di ciò che è oscuro e del tutto pertinente. Quelli, se Catone, Pisone, Torquato, Lucullo discuteranno di filosofia nell'opera di Cicerone, raccoglieranno sì molto materiale, ma lo confonderanno in modo tale che esso sembrerà non avere né capo né coda; questo libro, invece, spiegherà il più chiaramente possibile perché parlino Catone, Pisone, Torquato, Lucullo invece che Varrone, Cotta, Balbo e Triario, e perché parlino in quel modo e non in un altro [...] Insomma: qualunque cosa nell'opera di

44 Corradi 1552, p. n. n. (= II della premessa al lettore): Videremus in eum librum, tanquam in equum Troianum, quum Graecos tum Latinos oratores a Cicerone ita fuisse congestos, ut, qui bene norit illos, omne paene Ciceronianas personas cognosse videri possit.

45 Cf. Corradi 1544, f. 3r.: De propiis nominibus, quum doctorum hominum diversas opiniones esse videremus, quod alii ne quaerenda quidem putarent, alii vero epistolas hasce a nemine posse interpretari crederent, nisi ab eo, qui historias omnes etiam privatorum hominum ut ungues nosset suos, nos, qui neque multum neque nihil operae dandum huic rei semper censuimus, nomina eorum, qui vel ita ignobiles fuere, vel ita reiecti a scriptoribus, vel recepti illi quidem, sed ita quum illorum libris desiderati, ut vix, ac ne vix quidem, inveniri posse viderentur, curiosis quaerenda reliquimus, praesertim si ea cognosse non magni referre putavimus.

46 Corradi 1552, p. n. n. (= I-II della premessa al lettore). 
Cicerone riguardi la storia, la filosofia, l'arte oratoria, questo libro ce lo spiegherà, se solo vorrà dedicare a questo compito tanto impegno quanto è richiesto, in modo tale che non possiamo desiderare nulla di più.

Le preoccupazioni espresse dal Corradi si inseriscono perfettamente nell'umanesimo della metà del Cinquecento, intenzionato a ricostruire minuziosamente lo sfondo storico e culturale delle opere antiche. Se centro propulsore di tale orientamento era stata la Venezia primo-cinquecentesca, esso verrà ulteriormente valorizzato a Bologna, alla cui cattedra di umanità si succederanno per tutto il sedicesimo secolo studiosi - lo stesso Corradi (1544-1556), Francesco Robortello (1557-1561), Carlo Sigonio (1563-1584), Aldo Manuzio il Giovane (1585-1586) - formatisi nella città lagunare, ma che proprio con la docenza presso l'Alma Mater e le correlate pubblicazioni produrranno i migliori frutti in tal senso. ${ }^{47}$

\section{La biografia di Cicerone nella Quaestura}

L'esito più maturo di tale prospettiva è avvertibile nella Quaestura del 1555, dove l'interesse per la figura di Cicerone si estende a tutti gli aspetti correlati che possono contribuire a illuminarne ulteriormente il contesto e la vicenda storica: l'opera incontrò non a caso larga fortuna in epoche animate da zelo antiquario, tanto da essere ristampata nel 1754 a Lipsia per le cure di uno dei maggiori antichisti di area germanica dell'epoca, Johann August Ernesti. ${ }^{48}$ Oltre alla biografia di Cicerone, la Quaestura contiene, come indicato nel sommario iniziale, la cronologia delle sue opere pervenute e perdute (p. 286-310), ricostruita sulla base di testimonianze dello stesso Cicerone e di fonti successive, la biografia del figlio Marco (p. 310 -313), del fratello Quinto e di suo figlio (p. $314-$ 321), un'analisi del Commentariolum petitionis (p. 321-337) e un'ampia discussione dell'Invectiva in Ciceronem (p. 96-120) e dell'Invectiva in Sallustium (p. 121-143), di cui Corradi confuta, tra i primi, le rispettive paternità sallustiana e ciceroniana. ${ }^{49}$ L'esigenza di calarsi quanto più possibile nella realtà storica romana, comprendendone a fondo gli aspetti sociali e istituzionali, si riflette nell'inedito impianto dell'opera, nella quale i tre protagonisti, vestendo i panni

47 Sugli studi classici connessi con la cattedra di umanità dell'Alma Mater dell'epoca si vedano Costa 1907; Sorbelli/Simeoni 1987, II 42-47; Calcaterra 2009, 239-252.

48 Corradi 1754. L'edizione contiene anche la prima Quaestura del 1537.

49 Il ruolo del Corradi nel dibattito sulla paternità delle due invettive è ricordato in Novokhatko 2009, 116 - 117; sulle due opere si veda anche Degl'Innocenti Pierini (p. 61-81) in questo volume. 
di magistrati della Roma repubblicana e riproponendo dinamiche delle istituzioni antiche, si fingono contemporanei di Cicerone e inscenano il momento in cui al questore latino era richiesta dinanzi ai consoli la rendicontazione dei tributi riscossi:

Cor: Venetias profectus, illum [sc. Egnatium], Ioannemque Pierium Valerianum, quum Consules etiam tunc essent et de Republica, cuius aerarium videbant exhaustum, loquerentur, conveni: qui me quum vidissent, benigneque et comiter, ut ante solebant, excepissent, quum salvum venisse gavisi sint, tum divinitus esse factum crediderint, ut is, qui Quaestor esset, eo potissimum tempore, quo de Reipublicae rationibus ipsi commentabantur, adveniret. Itaque me rogare, vel potius urgere, coeperunt, ut Quaesturae rationem, quam prius inchoassem, aliquando tandem totam referrem.

Cor.: Partito per Venezia, raggiunsi lui [sc. l'Egnazio] e Giovanni Pierio Valeriano, quando allora erano ancora Consoli e parlavano dello Stato, il cui erario vedevano esausto; i due, dopo avermi visto e accolto con affetto e affabilità, come già erano soliti fare, si rallegrarono che io fossi giunto sano e salvo e credettero che accadesse per volere divino il fatto che chi era Questore sopraggiungesse proprio in quel tempo in cui loro discutevano dei conti dello Stato. E così cominciarono a chiedere, e anzi a incalzare, che io finalmente una buona volta riferissi tutto il bilancio della Questura che avevo precedentemente intrapreso.

La natura allegorica della situazione è trasparente: la Repubblica non è più quella romana, bensì la Respublica litterarum; i suoi massimi magistrati, i Consoli, sono gli anziani esponenti dell'umanesimo veneziano, l'Egnazio e il Valeriano, mentre il più giovane Corradi, ancora agli inizi del cursus honorum, è il questore che lascia la capitale degli studi, dove però saltuariamente torna, per svolgere il suo insegnamento nella provincia assegnatagli, Reggio Emilia; l'erario statale è il patrimonio di opere esistenti sulla figura di Cicerone, un tesoro bisognoso di essere rimpinguato per mezzo delle risorse riscosse dal Corradi, ossia grazie ai frutti delle sue ricerche e dei suoi insegnamenti, che una volta depositate nelle casse pubbliche saranno redistribuite ai poveri, cioè pubblicate e rese disponibili agli indotti e ai desiderosi di apprendere. ${ }^{50}$ L'originalità della cornice entro cui il discorso viene inserito è evidente sin dal titolo dell'opera, giocato sull'ambiguità del termine quaestura, che rimanda all'esazione dei tributi connessa con l'istituzione romana e all'indagine (da quaerere,

50 Corradi 1555a, 48: Cor.: Pauperibus ego mihi solvere videor, quum vobis solvo, qui nunc eius Reipublicae personam sustinetis, ex cuius aerario pauperes propemodum innumerabiles aluntur. Egn.: Qui sunt ii pauperes? Cor.: Indocti, qui cupiunt, vel, etiam si non cupiunt, interdum tamen possunt doceri. 
«ricercare») promossa nel dialogo. ${ }^{51}$ La consegna dei tributi riscossi, che comprende in misura minore monete straniere, cioè notizie ricavate da autori non latini, ${ }^{52}$ contribuisce a lumeggiare, attraverso la biografia di Cicerone, anche la vita e l'opera di altre personalità a lui variamente legate, con il risultato che «rinasceranno» uomini cancellati dal tempo o dalla trascuratezza degli studiosi.

La polemica contro la negligentia dei precedenti scrittori, già affacciatasi nella premessa del commento al Brutus, riaffiora con ulteriore vigore nella Quaestura, il cui dichiarato obiettivo è quello di riaffermare una serie di verità storiche, circa Cicerone e il suo tempo, contro le falsità presenti in Plutarco, Dione, Appiano e altri (Plutarchus, Dion, Appianus et alii tam multa falso retulerunt, p. 15). Reale bersaglio polemico del Corradi è l'atteggiamento ostile a Cicerone, o comunque il ritratto negativo che emerge a più riprese nell'opera dei predecessori moderni e antichi, evidentemente inaccettabile in un contesto che aveva elevato l'Arpinate a suprema autorità stilistica, morale e sapienziale: il vero obiettivo della Quaestura è infatti un'accorata difesa di Cicerone, come appare evidente nell'indice, dove si annuncia la Ciceronis vita undique collecta et defensa; l'opera mira in sostanza a ristabilirne il buon nome anche sotto quegli aspetti per i quali l'Arpinate era stato screditato nella tradizione biografica, senza implicare una falsificazione del dato storico da parte del Corradi, ma certo comportandone un uso tendenzioso. Dell'impostazione sostanzialmente panegiristica dell'opera si accorgerà un altro biografo di Cicerone, l'inglese Conyers Middleton, che nella sua The History of the Life of M. Tullius Cicero (1741), pur riconoscendo l'immutata utilità della Quaestura, la sua vasta informazione, la generale fondatezza dei giudizi e la sua eleganza stilistica, non mancherà di rilevarne la prospettiva integralmente filo-ciceroniana:

There are two books, however, which have been of real use to me, Sebastiani Corradi Quaestura and M.T. Ciceronis Historia a Francisco Fabricio: the first was the work of an Italian Critic of eminent learning, who spent a great part of his life in explaining Cicero's writings; but it is rather an apology for Cicero, than the History of his life; it's chief end being to vindicate Cicero's character from all the objections, that ever been made to it, and particularly from the misrepresentations of Plutarch and the calumnies of Dio. The piece is

51 Corradi 1555a, 6: Cor.: Hic noster [sc. dialogus], quum de magnorum virorum moribus et vita, tum de numis, quibus Respublica servari solet, habetur, et de rebus hisce disputando, vel potius exigendo, quaerit ita ut vel ob id Quaestura dicatur.

52 Corradi 1555a, 17-18: Egn.: Cogitat hic homo non Romanos solum et veteres, ut pollicitus est, sed externos etiam numos, dare. Cor.: Id vestra refert nihil, dum sint probi, quanquam sunt ii quidem omnes fere Ciceroniani et ita Romani; fieri tamen potest, ut externi etiam multi sint admisti, qui si in manus venerint, non credo vos propter legem latam reiecturos. Egn.: Bene credis, nisi fuerint reiiciendi. 
learned and ingenious, and written in good Latin; yet the dialogue is carried on with to harsh and forced an Allegory, of a Quaestor or Treasurer producing the several testimonies of Cicero's acts, under the form of genuine money, in opposition to the spurious coins of the Greek historians, that none can read it with pleasure, few with patience: the observations, however, are generally just and well-grounded, except that the Author's zeal for Cicero's honour gets the better sometimes of his judgment, and draws him into a defence of his conduct where Cicero himself has even condemned it. ${ }^{53}$

Lo scandaglio critico delle fonti, riferito sin dall'indice (multa e Plutarcho caeterisque Graeca conversa. Multa comprobata. Multa confutata), pone al centro la sola biografia ciceroniana completa sopravvissuta dall'antichità, quella di Plutarco. Il primato d'importanza dell'opera, dovuto a questa sua unicità, riflette peraltro un interesse particolarmente vivo nella Bologna del tempo grazie alla traduzione latina della vita plutarchea pubblicata nel 1508 dall'allora lettore di greco dello Studio cittadino Achille Bocchi (1488-1562), ricordato per questo suo lavoro anche nel Ciceronianus erasmiano. ${ }^{54}$ Personalità di rilievo nella cultura bolognese, nel 1546 Bocchi aveva fondato l'Hermathena, un'accademia patrocinata dal cardinale Alessandro Farnese e frequentata dalle maggiori personalità della cultura cittadina; con Corradi, che lo definisce noster amicus e vir nobilissimus (p. 44) e cita passi della sua traduzione, egli condivideva anche interessi ciceroniani, testimoniati da tuttora inedite Praelectiones a un corso sul De legibus del $1557 . .^{55}$ La completezza cronologica della vita plutarchea permette al Corradi di seguirne l'ordine di esposizione, riportandone in traduzione latina interi passi trascurati o non bene intesi da precedenti interpreti, specialmente dal Bruni, ${ }^{56}$ benché egli presenti l'opera del greco come una trama da «ritessere» in più punti, in quanto fonte lacunosa ed erronea (p. 35). Mentre l'Egnazio ravvisa in Plutarco uno scrittore equo e non partigiano, come si conviene a un biografo, Corradi ne denuncia la parzialità, includendolo nella folta schiera di quanti hanno dato di Cicerone un'immagine pregiudizialmente negativa, tralasciando i pur numerosi motivi di elogio di cui avrebbero dovuto dare conto (p. 158-159):

Egn.: Meminisse debes eos, qui vitas scribunt, oportere non virtutes modo, sed etiam vitia, quaecunque fuerint illa, complecti. Cor.: At hic ille [sc. Plutarchus] multa, quae laudari,

53 Middleton 1741, I, xxx-xxxi; sull'opera e l'autore si veda, oltre a Kenty (p. 205-206) e Berno (p. 370) in questo volume, Ingram 2015.

54 Cf. Gambaro 1965, 216; la traduzione è Bocchi 1508.

55 Per queste ed altre informazioni su Achille Bocchi cf. Rotondò 1969.

56 Corradi 1555a, 47: Haec verba nos idcirco vertimus, quod ab aliis ea vel male conversa, vel omissa prorsus videamus. Nam Leonardus Arretinus neque sensum more suo, neque verba convertit, vel quod ea in suo libro non invenerit, vel quod indigna putarit, quae a Plutarcho et de Cicerone scripta dicantur. 
certe referri debebant, tacuit; unum quod vituperari posse credidit, multis etiam verbis est persecutus. [...] Graecos omnes, quum caeteris Latinis, tum Ciceroni, qui primus eorum luminibus officere coepit, iniquissimos fuisse.

Egn.: Devi ricordare che quanti raccontano le vite bisogna che trattino non solo le virtù, ma anche i vizi, di qualunque natura essi siano. Cor.: Ma qui egli [sc. Plutarco] tacque molte cose che senz'altro dovevano essere riferite e lodate, mentre affrontò con molte parole soltanto ciò che ritenne potesse essere biasimato. [...] Tutti i Greci sono stati ingiustissimi non solo verso tutti i Latini, ma soprattutto verso Cicerone, che per primo cominciò a offuscare il loro lustro.

Persino il capitolo plutarcheo dedicato al proconsolato di Cicerone in Cilicia (Cic. 36), che restituisce un'immagine assolutamente lusinghiera dell'Arpinate, appare al Corradi inadeguato, perché troppo sbrigativo rispetto alla più ampia trattazione che i meriti e i successi di Cicerone avrebbero meritato, e che viene integrata dallo stesso Corradi sulla base dell'epistolario dell'oratore. ${ }^{57} \mathrm{Il}$ giudizio negativo su Plutarco investe anche gli altri autori greci che forniscono informazioni sulla vita di Cicerone, quali Dione e Appiano, tutti tacciati di non costante affidabilità e perciò in genere svalutati rispetto alle fonti latine: di Dione, in particolare, si dice che «mente spesso con l'intenzione di denigrare Cicerone» (studio de Cicerone detrahendi saepe mentitur, p. 24), e le falsità da lui raccolte nel discorso di Caleno sono talmente palesi da essere indegne di confutazione. ${ }^{58}$ La critica generalizzata alla fonti greche, che suscita la perplessità dell'Egnazio, viene giustificata dal Corradi in relazione al racconto della vicenda di Catilina, rispetto alla quale anche autori latini stringati e ineleganti appaiono preferibili agli autori greci, magari stilisticamente migliori, in quanto più attenti alla meticolosa ricostruzione dei fatti, con il metaforico risultato che le monete dei Latini, piccole ma di oro puro, vengono guastate dal bronzo mischiatovi dai Greci per renderle più grosse e appariscenti (p. 92-93):

Cor.: Qui numos diligenter spectant et bene probant, eos [sc. Paterculi] malunt, ita circuncisos, quam quos Diodorus Siculus, Plutarchus, Dion, Appianus et alii Graeci quam maximos formarunt. Pie.: Quid ita? Cor.: Quod illi, dum numos veteres probos illos quidem,

57 Corradi 1555a, 215: Egn.: Si quid ante Plutarchus, ut saepe questus es, detraxit, id hoc elogio quasi plena manu reddidit ita ut amplius queri non possis, eum, quum de Ciceronis laude scriberet, parcum fuisse. Cor.: Gratias agit et rem, quae fuerit omnium iudicio et testimonio probata, refert et tamen eam parcius multo, quam fuit aequum, describit. Nam res a Cicerone tunc in Cilicia gestas, quae vel longo commentario fuerant explicandae, paucis versibus ipse complectitur.

58 Corradi 1555a, 272: Cor.: Dion, qui res ita contrarias, ita leves, ita plane falsas scribat, ut facile quivis intelligat eas ne dignas esse quae confutentur. Nos certe, quamvis eas nullo negotio possimus, nolumus tamen confutare, ne tempus ipsum frustra conteramus. Sulla presenza di Cicerone in Cassio Dione cf. Gowing 1998 e Montecalvo 2014. 
et ex optimo aureo factos, sed nimis, ut ipse dicis, circuncisos, renovare ac reficere conantur, tantum aeris admiscuerunt, ut eos nemo amplius aureos esse dicat. Egn.: Nos dicimus, qui scimus a Plutarcho paene nihil expressum, quod auro non sit etiam purius. Cor: Ita vobis videri potest, sed mihi neque Plutarchus, neque alius quispiam Graecus, praeter Polybium et Dioysium, satis in ea re facit [...] Egn.: Tu malis numos Flori, Orosii, Eutropii, Rufi et aliorum, qui vix latine loquuntur, et, dum breves esse laborant, obscuri et negligentes fiunt, quam Graecorum, qui et elegantissime graece scripserunt et minima quaeque diligentissime sunt persecuti? Cor: Vide quam diligenter: saepe quae nusquam gentium sunt facta scribunt; saepius tempora, magistratus et magistratuum atque privatorum nomina confundunt; saepissime in his, quae facta sunt, more suo mentiuntur.

Cor:: Quanti valutano attentamente e soppesano bene le monete, preferiscono queste [scil. quelle di Velleio Patercolo], così minute, a quelle molto grandi che produssero Diodoro Siculo, Plutarco, Dione, Appiano e altri Greci. Pie.: Perché? Cor.: Perché questi, tentando di rifare e riparare le antiche monete, autentiche e fatte di ottimo oro, ma, come dici tu, troppo minute, vi hanno mischiato tanto bronzo che nessuno direbbe più che esse siano di oro. Egn.: Lo diciamo noi, che sappiamo che da Plutarco non è stato prodotto nulla che non sia anche più puro dell'oro. Cor.: A voi può apparire così, ma a me sotto questo aspetto non soddisfa né Plutarco né altro greco, eccetto Polibio e Dionigi di Alicarnasso [...] Egn.: Tu preferisci le monete di Floro, Orosio, Eutropio, Rufo e di altri, che a stento parlano latino, e, mentre si impegnano a essere brevi, divengono oscuri e sciatti, rispetto ai Greci che non solo hanno scritto con somma eleganza in greco, ma hanno anche curato con somma attenzione tutti i minimi dettagli? Cor.: Guarda con quanta attenzione: spesso scrivono cose che non sono accadute in nessun luogo; più spesso confondono tempi, cariche, nomi di magistrati e di privati cittadini; spessissimo mentono, come è loro costume, sui fatti accaduti.

La centralità di Plutarco per chiunque voglia scrivere la biografia di Cicerone era implicitamente riconosciuta già dal Bruni, il quale, proprio nel ricondurre l'idea di comporre il Cicero novus all'insoddisfazione per quanto contenuto nella vita plutarchea, confessava però che l'intento iniziale era stato quello di tradurre in latino l'opera. ${ }^{59}$ L'influenza esercitata da Plutarco sul Bruni viene rilevata dal Corradi, che perciò non risparmia critiche anche all'Aretino, perché per molti aspetti Plutarco fu per lui come un caecus dux per un cieco, tanto che obiettivo dichiarato della Quaestura è riuscire a «vedere dove Plutarco e il Bruni non hanno saputo vedere» (p. 19).

L'atteggiamento decisamente favorevole a Cicerone non è del resto taciuto dal Corradi, che fornisce ampio materiale («molte monete») volto a restituirne un'immagine positiva, dichiarando apertamente la propria felicità per il suo acclamato e trionfante ritorno dall'esilio (p. 202). L'innegabile parzialità della prospettiva si riflette, da un lato, nella condanna senza appello delle fonti ostili a Cicerone, come l'Invettiva pseudo-sallustiana, prodotto di un mediocre decla-

59 Cf. Viti 1996, 416-418. 
mator a cui si rifà Dione e di cui Corradi dimostra il carattere falso e diffamatorio, ${ }^{60}$ e dall'altro nell'ampio ricorso alla stessa opera ciceroniana come fonte autobiografica: quest'ultima scelta, dall'Egnazio giudicata rischiosa, perché ognuno non può che fornire un ritratto interessato di se stesso, ${ }^{61}$ è invece difesa da Corradi come assolutamente logica, tanto più a causa della perdita delle biografie ciceroniane cronologicamente più vicine al protagonista, ossia quelle di Nepote, Tirone e Fenestella (p. 19).

Notizie prive di riscontro nell'opera ciceroniana vengono così rigettate come false, come per esempio l'accusa, riferita da Plut. Cic. 8.1, secondo la quale Cicerone si sarebbe fatto corrompere per ridurre l'entità della sanzione a Verre: secondo Corradi la notizia è infondata, perché, se davvero Cicerone fosse stato accusato di corruzione, non avrebbe mancato di scagionarsi in qualche passo della propria opera. ${ }^{62}$ Il ricorso agli scritti ciceroniani appare a maggior ragione necessario in relazione agli argomenti e ai periodi sui quali le biografie tacciono, mentre in caso di discordanza tra le fonti il credito viene immancabilmente accordato alla testimonianza ciceroniana: il confronto è particolarmente serrato con la vita di Plutarco, che viene corretto in tutti i punti in cui discorda da Cicerone, mentre viene apprezzato quando mutua le informazioni direttamente da lui (come nel caso del racconto sulla vicenda di Catilina), ${ }^{63}$ o quando (troppo di rado secondo Corradi) ${ }^{64}$ ne offre un'immagine positiva.

Il ritratto intensamente apologetico, e anzi celebrativo, che Corradi offre dell'Arpinate emerge con particolare evidenza in riferimento a circostanze nelle quali il suo comportamento viene da sempre fatto oggetto di critiche e accuse

60 Corradi 1555a, 112: Suspiciose et criminose declamatorio more dicebas [sc. Ps. Sallustius], pro quibus te nunc etiam gravissimas poenas apud Inferos dare credimus, qui viro sanctissimo non solum turpitudine male dixeris ipse, sed etiam causa fueris ut alii maledicerent, ut Dion, qui sic ista maledicta collegit et te sic imitatus est ut simia quaedam fuisse videatur.

61 Cf. e.g. Corradi 1555a, 53, in relazione alla discrepanza tra Plut. Cic. 6.1 e Cic. Planc. 64-65 circa le reazioni alla questura ciceroniana in Sicilia: Egn.: Hic ego Plutarcho potius, quam Ciceroni credendum puto: quod hic de se loquens in eo praesertim, quod ad laudem pertinet, fortasse mentiatur, ille, ut historicus, quod verum sit, scribat, et causas addat cur molestus esset. 62 Corradi 1555a, 64: Cor.: Quod si ea unquam fuisset opinio, aliquis aliquando adversarius Ciceroni obiecisset. Egn.: Obiecerunt fortasse multi, sed scripta non extant in quibus id cognosci possit. Cor.: At ipsius Ciceronis extant orationes, in quibus quum reliqua, quae sunt obiecta, confutet, id quoque, si obiectum fuisset, aliqua ratione confutaret.

63 Cf. e.g. Corradi 1555a, 149: Cor.: In quattuor libris, quos Cicero contra Catilinam scripsit, unde Plutarchus, quicquid de coniuratione bene scripsit, est mutuatus.

64 Corradi 1555a, 195: Cor.: Videtis iam quod omnes vident, Plutarchum, qui de nugis saepe tam multa scripsit, in hisce rebus, quae ad gloriam Ciceronis pertinent, ita parcum fuisse, vix ut eas indicarit. 
nella tradizione biografica. ${ }^{65}$ Uno dei momenti più controversi della vita di Cicerone è lo scoppio della guerra civile tra Cesare e Pompeo, quando già i contemporanei e poi i posteri lo accusarono di atteggiamento ambiguo e incerto (Att. 9.1.3), sospettandone addirittura un'iniziale collocazione filo-cesariana per il fatto che sulle prime egli non seguì Pompeo lontano da Roma dopo il passaggio del Rubicone (Plut. Cic. 37.2-3). L'eventuale indecisione di Cicerone nello schierarsi viene negata dal Corradi, preoccupato soprattutto di scagionarlo dal sospetto più inquietante, ossia quello di aver parteggiato per Cesare: Cicerone aveva in realtà deciso sin da subito di schierarsi con Pompeo, secondo quanto si ricava dalle sue lettere ad Attico, in particolare da Att. 7.3.5, fonti dal Corradi giudicate attendibili perché non si tratta di opere scritte con intenti apologetici, ma di corrispondenza privata nella quale il mittente ha l'urgenza di condividere il da farsi con un amico fidato; la sua posizione fu però cauta e poco esibita perché egli tentò in ogni modo di invitare i due contendenti alla pace, da lui auspicata più di ogni altra cosa (Att. 8.2.1), e perché un'esposizione troppo aperta al fianco di Pompeo, se i due contendenti si fossero poi riconciliati, avrebbe provocato le ritorsioni di Cesare (Att. 8.11d. 7-8); quando Pompeo decise di lasciare Roma, Cicerone aveva già deciso di seguirlo, ma dissimulò le proprie intenzioni per timore di essere ostacolato nei suoi progetti. ${ }^{66}$ Alla perplessità dell'Egnazio, disposto a concedere che inizialmente Cicerone parteggiasse per Pompeo, ma convinto che avesse poi cambiato idea più volte, Corradi replica spiegando che l'Arpinate ponderò con cura la situazione, domandandosi (Att. 8.7.2) se il Pompeo di un tempo, garante della tradizione repubblicana, fosse ancora lo stesso Pompeo, che respingeva le condizioni di pace ed era ormai pronto a tutto (Att. 8.8.1). Il suo dubbio, in sostanza, non riguardò mai quale comandante seguire, ma se seguire Pompeo o rimanere neutrale. ${ }^{67}$

65 Per la presenza di simili accuse, e in parte anche delle difese addotte da Corradi, già in epoca antica si vedano in questo volume Stoner (p. 83-99), in relazione a Quintiliano, e La Bua (p. 103-118), in relazione agli storici antichi.

66 Corradi 1555a, 225: Cor.: [sc. Scio] Ciceronem iam inde a principio consituisse in bello Pompeium sequi nec unquam postea mutasse sententiam: sed quum videret agi de pace et professionem talis voluntatis esse sibi periculosam, cunctatum et tergiversatum esse, donec Pompeius ex Italia fugit: tum vero semper de discessu cogitasse, sed occultasse consilium, ne posset vel ab Antonio vel ab aliis impediri. Egn.: Si tu ista probares, nemo posset amplius dicere Ciceronem tum mobilem vel mutabilem fuisse. Cor.: Nos, inquam, vel ex epistolis ea scire possumus. Egn.: Quid si suspectae sunt epistolae? Cor.: Ille non possunt esse suspectae, quas ille tunc ad Atticum scripsit, non ut se defenderet, sed ut illum de suis consiliis admoneret, quicum solebat omnia libere communicare.

67 Corradi 1555a, 226-227: Quid opus erat deliberare, si iampridem Pompeium sequi constituerat? Cor.: Illum Pompeium, qui prius erat, sequi constituerat, sed deliberabat, an eum Pompeium, 
L'atteggiamento incostante dell'Arpinate si sarebbe rivelato anche durante il suo esilio, come riferito in Plut. Cic. 32.5, ma secondo Corradi si tratta di una calunnia, né può essere addotto come prova il tono supplichevole e lamentoso delle lettere ciceroniane, perché esso è piuttosto una posa dettata dal tentativo di esortare i familiari a perorare la sua causa (p. 186):

Cor: Videtis, opinor, ut homo Graecus, omnes occasiones, ut Ciceroni maledicere possit, captet. Egn.: Non captaret ille, nisi Cicero, qui tam miserabiliter ad Atticum, ad Quintum fratrem et ad Terentiam scribat, offerret. Cor.: Ille vero miserabiliter scribebat ad eos, ut miserabilis videretur, quo magis eos ad id procurandum, quod cupiebat, excitaret.

Cor: Vedete, credo, come un uomo greco approfitti di ogni occasione per poter denigrare Cicerone. Egn.: Ma non ne approfitterebbe se non la offrisse Cicerone, che scrive in maniera tanto lamentosa ad Attico, al fratello Quinto e a Terenzia. Cor.: In realtà scriveva loro in maniera lamentosa per apparire in una condizione miserevole e sollecitarli maggiormente a ottenere ciò che desiderava.

Cicerone è così restituito alla piena dignità del sapiens, capace di affrontare le tempeste della vita con saldezza d'animo e coraggio, in ciò distinguendosi da Demostene, che «compiangeva il proprio esilio con modi da donna» (muliebriter exilium suum deflebat, p. 187). La questione si intreccia con un altro difetto ampiamente rimproverato a Cicerone dalla tradizione biografica, ossia la sua codardia: il motivo, rinfacciatogli sin da Plut. Cic. 19.5, sarà continuamente riproposto fino al Novecento, quando nel 1939 Alex F. Witley intitolerà la propria biografia ciceroniana The Tremulous Hero. Secondo Plut. Cic. 4.6, la viltà di Cicerone si sarebbe palesata già quando, subito dopo il successo forense ottenuto con la Pro Roscio, l'oratore lasciò Roma alla volta di Atene per timore di Silla; la notizia, accolta dal Bruni, ${ }^{68}$ viene smentita dal Corradi sulla base di Brut. 313-314, dove Cicerone afferma di aver continuato a trattare cause a Roma per altri due anni prima di partire (nel 79 a. C.), sconfessando nei fatti, con il proprio impegno pubblico, la vociferata paura di Silla. La pusillanimità di Cicerone si sarebbe poi manifestata al massimo grado in occasione del processo a Milone, quando secondo Plut. Cic. 35.3-5 e Dio Cass. 45.54.2 l'oratore sarebbe stato così intimorito dalla presenza dei soldati pompeiani nel foro da non riuscire a pronunciare che un'orazione flebile e singhiozzante, tanto che Milone fu condannato: benché lo stesso Cicerone, in Mil. 1, confessi di rimanere impressionato alla vista dei soldati, l'ardore apologetico induce Corradi a ipotizzare che a terro-

qui tunc esset, sequeretur [...] Quamvis ille nunquam deliberarit utrum ducem, sed an Pompeium an neutrum sequeretur. Sul posizionamento di Cicerone nella guerra civile si veda comunque Scardigli 1995, 524-529 con note.

68 Cf. Viti 1996, 422. 
rizzare l'oratore fu più che altro il fragore dei Clodiani (p. 211), basando l'argomentazione su Cic. Mil. 3, fam. 3.10.10 e Att. 9.7b.2, dove l'Arpinate descrive interessatamente la presenza dei soldati pompeiani non come una minaccia, bensì come un presidio in sua difesa contro la massa inferocita dei Clodiani. La difesa di Cicerone è comunque condotta dal Corradi non negando il timore da lui provato, ma con la dimostrazione che il timore non è un vizio da biasimare, come sostiene Crasso in de orat. 1.119-121 quando afferma che è anzi qualità del bravo oratore iniziare un discorso con una certa timidezza, e del resto lo stesso Cicerone (Caec. 41) confessa di essere percorso da un tremore fisico durante le arringhe, segno che l'eventuale paura da lui provata non può essere motivo di biasimo (tantum abest, ut ob eum timorem reprehendi possit aut debeat, p. 273). La condanna di Milone non è dunque da imputare alla pavidità ciceroniana, ma alla rinuncia dell'imputato alla supplica della giuria, riferita in Plut. Cic. 35.5, e all'ostilità di Pompeo nei suoi confronti, riferita in Vell. Pat. 2.47.4 (p. 212).

Corradi non ha dubbi nemmeno sul ruolo avuto da Cicerone nell'uccisione di Cesare: benché Plut. Cic. 42.1-2 escluda una sua partecipazione alla congiura, tornando a indugiare sulla sua natura di codardo, e lo stesso Cicerone dichiari di non essere stato nemmeno informato del progetto (Phil. 2.26), un suo ruolo sembra garantito da diversi indizi, quali la deprecazione del corso degli eventi instaurato da Cesare, che sembra tradursi in un'esortazione alla sua uccisione, l'accusa rivoltagli da Antonio di essere mandante morale dell'assassinio (Phil. 2.25) e la menzione del suo nome da parte dei congiurati (Phil. 2.28 e 30). Il ruolo di membro, se non ispiratore, della congiura, lungi dal rappresentare una macchia morale su Cicerone, appare al Corradi come motivo di ulteriore prestigio per un uomo che dimostrò ancora una volta di agire con coraggio nell'interesse della patria e in nome della libertas repubblicana calpestata dal tiranno (p. 250):

Cor:: M. Antonius dixit Caesarem consilio Ciceronis interfectum fuisse. Egn.: Id in Philippicis ipse negat. Cor.: Negat ille quidem, sed in Bruto sic, ipso Bruto praesente, civitatis cladem deplorat, ut illum prorsus ad liberandum Rempublicam invitare videatur. Quod si vel in scriptis id ipsum faciebat, quanto magis illum, quum secreto loqueretur, id fecisse putare debemus? Non sine causa, «interfecto Caesare, statim cruentum extollens alte M. Brutus pugionem, Ciceronem nominatim exclamavit, atque ei recuperatam libertatem est gratulatus» [Cic. Phil. 2.28]. Egn.: Nunc Ciceronem, quem semper defendere soles, accusas. Cor.: Ego vero, ne scriptor Graecus eum timidum, quod inter coniuratos numeratus non sit, appellet, defendo et laudo, quum Bruto, ut libertatem recuperaret, autorem fuisse contendo.

Cor:: Marco Antonio ha detto che Cesare era stato ucciso su consiglio di Cicerone. Egn.: Ma lo stesso Cicerone lo nega nelle Filippiche. Cor.: Lo nega, è vero, ma nel Bruto, alla presenza di Bruto stesso, deplora a tal punto la rovina della città che sembra davvero invitarlo a liberare lo Stato. E se si comportava così negli scritti, quanto a maggior ragione dobbiamo ritenere che abbia agito così quando parlava in segreto? Non senza motivo, «ucciso Cesare, 
subito Marco Bruto, alzando il pugnale insanguinato verso il cielo, gridò il nome di Cicerone e si congratulò con lui per la recuperata libertà» [Cic. Phil. 2.28]. Egn.: Ma ora accusi Cicerone, che sei solito difendere sempre. Cor.: Tutt'altro: io in realtà lo difendo e lo lodo, affinché lo scrittore greco non lo chiami pauroso per il fatto che non sarebbe tra i congiurati, dal momento che concordo fermamente con Bruto che egli fu il responsabile della recuperata libertà.

Se Cicerone nega di essere al corrente dell'azione, lo fa soltanto per non inimicarsi i sostenitori di Cesare e per non essere così escluso dalle trattative per la pacificazione interna allo Stato, anche perché un suo patente appoggio ai congiurati non sarebbe stato utile né alla sua persona né alla causa della Respublica.$^{69}$ Il suo comportamento nella vicenda dimostra così, agli occhi del Corradi, che egli non fu persona pavida, ma piuttosto uomo fortissimus (p. 251).

L'altro grande difetto, insieme alla pavidità, costantemente rimproverato a Cicerone nella tradizione biografica sin da Sen. brev. 5.1-3 e Plut. Cic. 24.1-3 è lo smodato narcisismo, che lo portava a elogiare continuamente se stesso, rendendosi così insopportabile ai contemporanei: oltre a citare il passo di Quint. 11.1.9 in cui l'Arpinate parla con modestia di sé e delle proprie capacità retoriche, Corradi giustifica l'abbondanza di autoreferenzialità nell'opera di Cicerone, perché questi loda le proprie azioni per rispondere agli avversari (p. 274), come lui stesso sostiene in dom. 93 e in har. resp. 16-17, dove ricorda che evocare le vicende personali significa raccontare le vicende dell'intera Respublica, e dunque di tutti i Romani. Il desiderio di gloria di Cicerone, peraltro da lui stesso ammesso (Att. 2.17.2), non lo distingue da molti altri personaggi della grecità e della romanità che vollero essere protagonisti dei propri stessi scritti e di quelli altrui; come per costoro si tratta di legittima ambizione, così questa abitudine non può costituire un motivo di biasimo di Cicerone, perché, lungi dall'essere prova di egocentrismo, essa manifesta piuttosto la consapevolezza della comune utilità che contemporanei e posteri potranno ricavare dalla lettura delle sue vicende (p. 274-276):

Cor.: qui reprehendunt [sc. Ciceronem], maximos quoque viros, ut Themistoclem, Alexandrum Magnum, Africanum, Pompeium et alios reprehendunt, qui nec minus ambitiosi fuerunt quam Cicero, nec minus quam ille res suas celebrari cupierunt, quum multi

69 Corradi 1555a, 250 -251: Egn.: Si fuisset [sc. autor cladis], ut erat gloriae cupidus, aliquando diceret. Cor.: Non erat stultus ut illi qui, quum in ea societate non fuissent, se tamen fuisse iactabant. Egn.: Stultus dici non posset, si se facti vel autorem vel participem fuisse diceret, quod ille pulcherrimum gloriosissimumque solet appellare. Cor.: Minus prudens certe videri potuisset, si diceret quod ipsi et Reipublicae damni multum, sed utilitatis aut nihil aut parum, poterat afferre. Nam si dixisset, veteranos milites caeterosque Cesarianos inimicos haberet, et ita non posset de pace tractare. 
scriptores ad id faciendum vel praemiis invitarint. [...] Xenophon, Aratus, Scaurus, Catulus, Caesar, Adrianus et alii res suas etiam scripserunt; ne quidam Ciceronem tam graviter, ut faciunt, accusent quod vel aliquid de se scripserit vel a Luceio, ut res suas scriberet, postularit. Quare si Ciceronem volunt accusare, illos etiam, quos diximus, et M. Varronem, C. Trebonium, qui a Cicerone, Plinium Iuniorem, qui a Cornelio Tacito, ac alios innumerabiles, qui ab aliis idem propemodum postularunt, accusent. [...] Res enim, quas gesserat, si scriberentur, non sibi solum, sed omnibus etiam hominibus, quum praesentibus tum futuris, etiam profuturas putabat; et idcirco, ut scriberentur et extarent, laborabat.

Cor.: quanti rimproverano [sc. Cicerone], rimproverano anche i più grandi uomini, come Temistocle, Alessandro Magno, l'Africano, Pompeo e altri, che non furono meno ambiziosi di Cicerone, né meno di lui desiderarono celebrare le loro mille imprese, ma anzi invitarono molti scrittori a farlo persino dietro ricompensa. [...] Senofonte, Arato, Scauro, Catulo, Cesare, Adriano e altri hanno raccontato le proprie imprese; dunque non accusino con tanta violenza, come fanno, nemmeno Cicerone per aver scritto qualcosa di sé o per aver chiesto di farlo a Luceio. Pertanto, se vogliono accusare Cicerone, accusino anche quelli che abbiamo citato, e Varrone, e Trebonio, che a Cicerone, e Plinio il Giovane, che a Traiano, e moltissimi altri, che ad altri, rivolsero sostanzialmente la stessa richiesta. [...] Infatti egli riteneva che le proprie azioni, se fossero state raccontate, avrebbero giovato non soltanto a lui, ma anche a tutti gli uomini, tanto ai contemporanei quanto ai posteri, e proprio per questo motivo si impegnava perché venissero raccontate e sopravvivessero.

Cicerone viene così scagionato da ogni biasimo, al punto che l'ecclesiastico Corradi, senza stridore con l'ormai avviato clima tridentino, approva senza problemi il fatto che l'Arpinate sia annoverato tra gli dei dall'imperatore Alessandro Severo, e, come se non bastasse, arriva addirittura a sostenere che Cicerone può essere legittimamente invocato contro le malattie agli occhi, richiamando l'epigramma (riportato in Plin. nat. hist. 31.8) del liberto ciceroniano Tullio Laurea in cui si magnificano le miracolose proprietà curative delle acque zampillanti presso una villa campana dell’Arpinate (p. 269-270):

Cor: Nihil mea refert, dum coelestem vocarit eum, cuius imaginem vel inter Divos, Alexander Severus Imperator Romanus, qui «Latina quum legeret, non alia magis, quam $D e$ officiis Ciceronis et De republica legebat» [SHA (Lampr. Alex. Sev.), 30.2], postea collocavit. Egn.: Quin igitur illi nos vota facimus? Cor.: Nos vero illi vota facimus, oculis ut nostris ille medeatur. Egn.: Oculis ut nostris ille, cui lippitudo tam saepe molesta fuit, medeatur? Cor.: Divi morbos, quibus dum viverent, affici solebant, in coelum sublati saepe curare creduntur. Aquae certe Ciceronianae, quas Tullius Laurea carmine celebravit, oculis, ut Plinius affirmat, medebantur.

Cor.: Non mi importa per nulla il fatto che abbia chiamato celeste Cicerone, la cui effigie collocò poi tra le divinità, l'imperatore Alessandro Severo, che «quando leggeva opere latine, non leggeva altro che il De officiis di Cicerone e il De repubblica» [SHA (Lampr. Alex. Sev.), 30.2]. Egn.: Suvvia, adesso noi arriviamo a fare voti a Cicerone? Cor.: Ma noi facciamo voti a lui, affinché curi i nostri occhi. Egn.: Affinché curi i nostri occhi proprio lui, a cui la congiuntivite diede sempre fastidi? Cor.: Ma si ritiene che gli dei, giunti in cielo, spesso 
curino proprio quelle malattie dalle quali erano affetti in vita. Sicuramente le acque ciceroniane, che Tullio Laurea celebrò in un carme, curavano gli occhi, come Plinio afferma.

Nel presentare come unico rimpianto il fatto che il vir sanctissimus (p. 112) Cicerone non abbia potuto conoscere il cristianesimo, Corradi perpetua però il già richiamato giudizio di ascendenza umanistica, risalente in prima istanza al Petrarca e ampiamente consolidato nella cultura dell'epoca, alla quale il paganesimo ciceroniano non appare un problema sostanziale, essendo la sua concezione religiosa pienamente conciliabile con la dottrina cristiana: «se Cicerone avesse conosciuto la nostra religione, non potremmo rimpiangere nulla in lui, tanto più che nemmeno su Dio ha avuto opinioni davvero sbagliate». ${ }^{70}$

Figura lontanissima dall'avvocato senza passione né convinzione dipinto da Mommsen, dal pusillanime narcisista tratteggiato da molta critica, specialmente a partire dall'Ottocento nel quadro dell'imperante cesarismo ${ }^{71}$ il Cicerone ritratto nella Quaestura soddisfa i consoli Egnazio e Valeriano, riconoscenti al questore Corradi per il denaro consegnato e sicuri che i conti ormai tornino: $:^{72}$ l'Arpinate viene presentato come il primo dei Romani, il salvatore della Repubblica e un fautore di pace, ${ }^{73}$ un uomo persino esteticamente irreprensibile, se un detrattore come Asinio Pollione parla in Sen. suas. 6.24 del suo aspetto decorso anche in vecchiaia (p. 282), insomma una personalità talmente straordinaria da non poter essere paragonata ad altro uomo esistito sulla faccia della terra (p. 283):

Cor.: Nec omnibus ego, sed singulis ita praefero, ut audeam paene dicere, a condito orbe neminem fuisse quem prorsus cum Cicerone conferre possimus.

70 Corradi 1555a, 280: Quod si Cicero nostram religionem cognovisset, nihil in eo possemus desiderare, quum praesertim nec de Deo admodum male senserit.

71 Sulla ricezione di Cicerone nella cultura ottocentesca cf. Narducci 2004, 277-363; una breve ma esemplare rassegna di giudizi critici sull'Arpinate in Scardigli 1995, 317-324.

72 Cf. la conclusione dell'opera in Corradi 1555a, 339: Egn.: Nos autem, quando tu iam totam Quaesturae rationem rationem retulisti et nos, qui pecuniam omnem in tabulas publicas referendam curavimus, videmus rationum summam quadrare, teque bene, et de Republica fecisse iudicamus, te in fano pecuniam iussu nostro deposuisse, si placet, quo tibi melius caveatur, ascribemus. Cor.: Placet.

73 Cf. Corradi 1555a, 117 (Cor.: Si Camillus, qui restituit, secundus Romulus est appellatus, cur Cicero, qui servavit imperium, non poterat vere Romulus appellari? Mihi tanto dignior eo nomine, quam Camillus, videtur, quanto maius eo tempore fuit imperium. Quin ego saepe mecum cogito, Ciceronem Romanis omnibus, qui Rempublicam bene vel domi vel foris unquam gesserunt, merito posse praeferri. Nam caeteri partes aliquas Reipublicae iuvarunt, sed eam Cicero solus universam conservavit) e 223 (Cicero vero semper auctor pacis fuit). 
Cor.: E per parte mia non a tutti genericamente, ma ad uno ad uno io preferisco Cicerone, al punto che oserei quasi dire che dalla nascita del mondo non vi fu nessuno che possiamo davvero paragonare a lui.

Se il fatto che i pareri sull'Arpinate divergano «come il giorno dalla notte è dovuto ai differenti pregiudizi, alle opinioni e all'atteggiamento dei vari autori, più che a una vera problematica legata ai fatti principali della sua vita», ${ }^{74}$ non potrà certo sorprendere che il ritratto di Cicerone al culmine dell'epoca del ciceronianismo sia, in fin dei conti, un ritratto agiografico.

74 Così Joseph Geiger nell'introduzione a Scardigli 1995, 293. 
